\title{
Optical emission spectroscopy of metal-halide lamps: Radially resolved atomic state distribution functions of Dy and $\mathrm{Hg}$
}

\section{Citation for published version (APA):}

Nimalasuriya, T., Flikweert, A. J., Stoffels, W. W., Haverlag, M., Mullen, van der, J. J. A. M., \& Pupat, N. B. M. (2006). Optical emission spectroscopy of metal-halide lamps: Radially resolved atomic state distribution functions of Dy and Hg. Journal of Applied Physics, 99(5), 053302-1/7. [053302].

https://doi.org/10.1063/1.2175466

DOI:

$10.1063 / 1.2175466$

Document status and date:

Published: 01/01/2006

\section{Document Version:}

Publisher's PDF, also known as Version of Record (includes final page, issue and volume numbers)

\section{Please check the document version of this publication:}

- A submitted manuscript is the version of the article upon submission and before peer-review. There can be important differences between the submitted version and the official published version of record. People interested in the research are advised to contact the author for the final version of the publication, or visit the $\mathrm{DOI}$ to the publisher's website.

- The final author version and the galley proof are versions of the publication after peer review.

- The final published version features the final layout of the paper including the volume, issue and page numbers.

Link to publication

\section{General rights}

Copyright and moral rights for the publications made accessible in the public portal are retained by the authors and/or other copyright owners and it is a condition of accessing publications that users recognise and abide by the legal requirements associated with these rights.

- Users may download and print one copy of any publication from the public portal for the purpose of private study or research.

- You may not further distribute the material or use it for any profit-making activity or commercial gain

- You may freely distribute the URL identifying the publication in the public portal.

If the publication is distributed under the terms of Article 25fa of the Dutch Copyright Act, indicated by the "Taverne" license above, please follow below link for the End User Agreement:

www.tue.nl/taverne

Take down policy

If you believe that this document breaches copyright please contact us at:

openaccess@tue.nl

providing details and we will investigate your claim. 


\title{
Optical emission spectroscopy of metal-halide lamps: Radially resolved atomic state distribution functions of Dy and $\mathrm{Hg}$
}

\author{
T. Nimalasuriya, ${ }^{\text {a) }}$ A. J. Flikweert, W. W. Stoffels, M. Haverlag, and \\ J. J. A. M. van der Mullen \\ Department of Applied Physics, Eindhoven University of Technology, P.O. Box 513, \\ 5600 MB Eindhoven, The Netherlands \\ N. B. M. Pupat ${ }^{\text {b) }}$ \\ École Nationale Supérieure de Chimie de Paris, 11 Rue Pierre et Marie Curie, 75005 Paris, France
}

(Received 22 August 2005; accepted 24 January 2006; published online 2 March 2006)

\begin{abstract}
Absolute line intensity measurements are performed on a metal-halide lamp. Several transitions of atomic and ionic Dy and atomic $\mathrm{Hg}$ are measured at different radial positions from which we obtain absolute atomic and ionic Dy intensity profiles. From these profiles we construct the radially resolved atomic state distribution function (ASDF) of the atomic and ionic Dy and the atomic $\mathrm{Hg}$. From these ASDFs several quantities are determined as functions of radial position, such as the (excitation) temperature, the ion ratio $\mathrm{Hg}^{+} / \mathrm{Dy}^{+}$, the electron density, the ground state, and the total density of Dy atoms and ions. Moreover, these ASDFs give us insight about the departure from equilibrium. The measurements show a hollow density profile for the atoms and the ionization of atoms in the center. In the outer parts of the lamp molecules dominate. (C) 2006 American Institute of Physics. [DOI: 10.1063/1.2175466]
\end{abstract}

\section{INTRODUCTION}

The search for a compact high-intensity light source with high luminous efficacy and good color rendering properties has led to the development of the metal-halide lamp. ${ }^{1}$ This type of lamp contains a buffer gas of $\mathrm{Hg}$ and a relatively small amount of a mixture of rare-earth additives such as Dy, $\mathrm{Ce}$, and $\mathrm{Na}$ salts, which act as the prime radiators. More than two salt components are necessary for a good color rendering index; therefore mixtures such as $\left(\mathrm{NaI}+\mathrm{ScI}_{3}\right),(\mathrm{NaI}+\mathrm{TII}$ $+\mathrm{InI})$, and $\left(\mathrm{NaI}+\mathrm{TlI}+\mathrm{DyI}_{3}+\mathrm{HoI}_{3}+\mathrm{TmI}_{3}\right)$ are commonly used in the metal-halide lamp. Because of radial diffusion and convection these additives are nonuniformly distributed over the lamp, resulting in the undesirable segregation of colors $^{2}$, (see Fig. 1). In this study a lamp with a Hg buffer gas and one salt, namely, $\mathrm{DyI}_{3}$, is used. This lamp has a relatively simple salt system, and therefore the results are easier to compare with a numerical model. Transport phenomena in this chemically complex plasma are not yet fully understood.

The aim of this work is to improve the understanding of the plasma properties and transport phenomena in this type of lamps. The lamp is investigated using absolute line intensity measurements. The intensity of the lines are calibrated using a ribbon lamp with known spectral radiance. The spectrum of Dy contains a large abundance of lines (see Fig. 2), making wavelength calibration a complicated task. After intensity and wavelength calibration, these measurements yield absolute radial density distributions of several excited states of the additive Dy and the buffer gas Hg. From these the atomic state distribution functions (ASDFs) are constructed

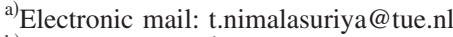

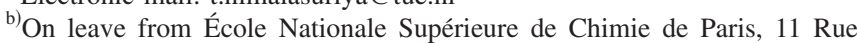
Pierre et Marie Curie, 75005 Paris, France.
}

for the atomic and ionic Dy and the atomic Hg. From the ASDFs several plasma properties can be determined, such as the temperature, the ground state densities, and the electron density. Moreover, the results may give an indication of a departure from equilibrium. This could be induced, for example, by transport phenomena such as segregation.

The few milligrams of the $\mathrm{DyI}_{3}$ additive do not entirely evaporate leaving a liquid salt pool at the coldest spot of the burner wall. The cold spot determines the vapor pressure of the additive in the immediate vicinity of the salt pool. Because of the large temperature gradient between the wall $(\sim 1200 \mathrm{~K})$ and the center of the burner $(\sim 5500 \mathrm{~K})$ (Ref. 3$)$

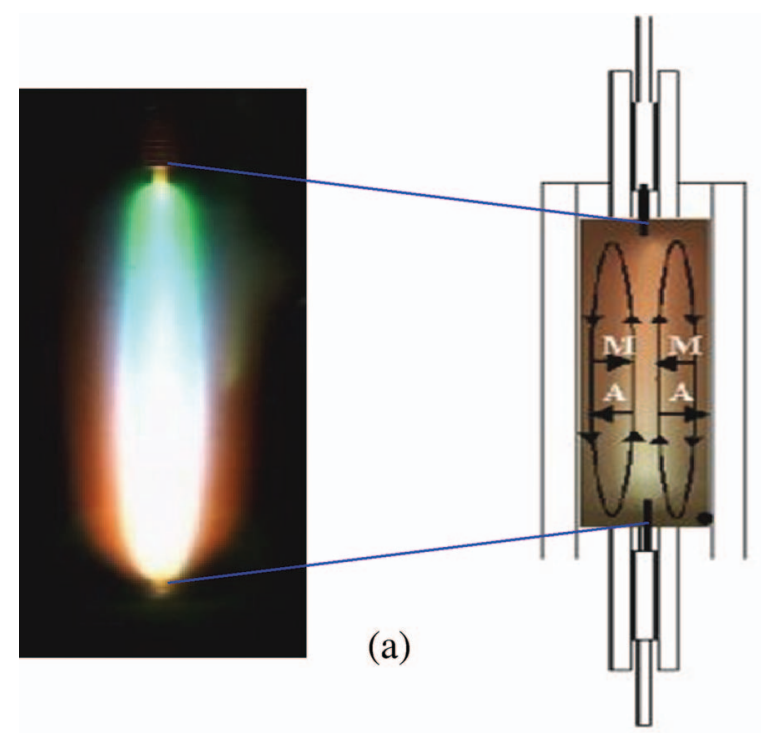

FIG. 1. (Color) (a) Color separation in a metal-halide (MH) lamp burner. (b) Schematic view of a MH lamp; diffusion and convection of atoms $(A)$ and molecules $(M)$ are indicated by arrows. 


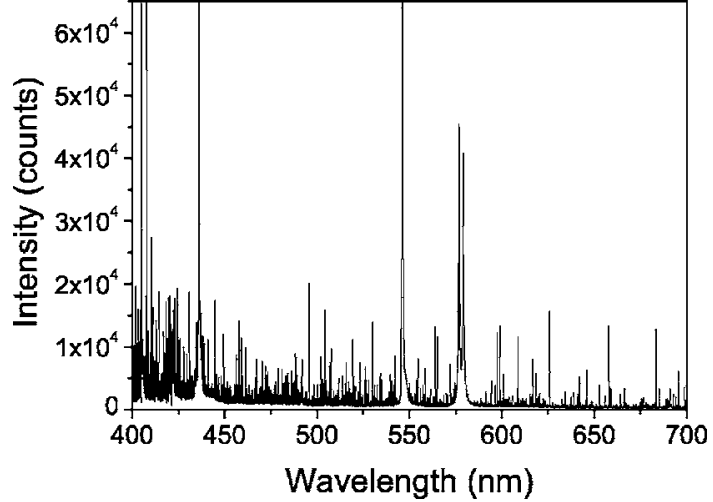

FIG. 2. Spectrum of a metal-halide lamp containing $\mathrm{Hg}$ as a buffer gas and the additive $\mathrm{DyI}_{3}$. The saturated lines are $\mathrm{Hg}$.

a multistep process of dissociation of $\mathrm{DyI}_{3}$ molecules towards the center and association of atoms into molecules near the wall takes place. The reaction chain can be described as follows, increasing with temperature to the right:

$$
\mathrm{DyI}_{3} \rightleftarrows \mathrm{DyI}_{2}+\mathrm{I} \rightleftarrows \mathrm{DyI}+2 \mathrm{I} \rightleftarrows \mathrm{Dy}+3 \mathrm{I} \text {. }
$$

At the hot center Dy atoms ionize and Dy ions are created,

$$
\mathrm{Dy}+e \rightleftarrows \mathrm{Dy}^{+}+e+e .
$$

Two mechanisms influence the distribution of particles in the plasma. First, there is a high temperature in the center rapidly decreasing toward the wall because of $p=n k T$. This results in a hollow profile of the mass distribution over the lamp. Second, there is difference between the diffusion velocities of the atoms and molecules. The smaller and lighter Dy atoms diffuse faster than the larger and heavier molecules (DyI, DyI ${ }_{2}$, and $\mathrm{DyI}_{3}$ ). This difference in diffusion velocity results, in a steady state, in an even more hollow profile of the elemental density of Dy; this is called radial segregation. Elemental density includes contributions from all molecular, atomic, and ionic species of a particular element.

Convection in the lamp causes the hot gas to move upwards in the center of the arc and downwards along the cool wall. This movement drags the high concentration of Dy near the wall downwards, resulting in a high density of elemental Dy in the bottom of the arc. This is called axial segregation. ${ }^{2}$ The combination of axial and radial segregations is shown in Fig. 1(b).

This paper is organized as follows. Sec. II deals with the theory of the atomic state distribution function and the absolute line intensity. Section III deals with the lamp and the experimental setup used for the optical emission spectroscopy measurements. In Sec. IV the atomic state distribution function for different radial positions and its derived quantities are presented and discussed. It includes the radial density profiles of the Dy atoms and ions and the $\mathrm{Hg}$ atoms, and the radial profiles of electron density and temperature. We also study the effects of the large presence of $\mathrm{Hg}$ on the plasma.

The results of this work will aid the validation of the numerical models meant to simulate transport phenomena in metal-halide lamps.

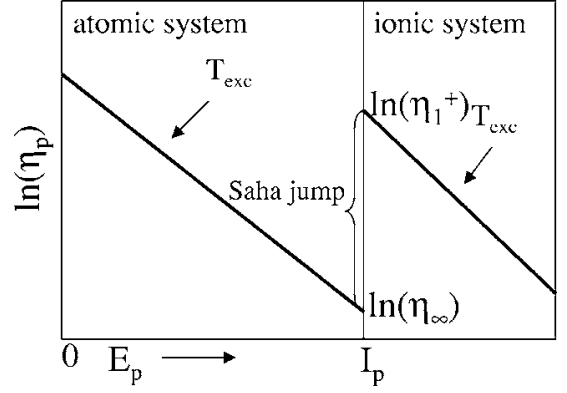

FIG. 3. The ASDF of two subsequent atomic stages of one element (e.g., Dy) for a plasma in LTE. At the transition from the atomic to the ionic system the state density $\eta$ shows a discontinuity. This is the so-called Saha jump and depends on the electron temperature and density [see Eq. (6)]. Note that there are two ways to indicate the energy position of an atomic level $p$ the excitation energy $E_{1 p}$ and the ionization potential $I_{p r}$. Going to the left along the energy axis, $I_{p r}$ will increase, whereas $E_{1 p}$ will decrease (Ref. 5).

\section{THEORY}

\section{A. The atomic state distribution function}

A plasma region is said to be in local thermal equilibrium (LTE) when, at a certain specific location, all the material particles are in equilibrium with each other. This means that for any collisional reaction the number of forward processes equals that of the corresponding backward processes. ${ }^{4}$ However, photons can, in principle, escape from a plasma region in LTE; therefore emission is not necessarily compensated by the inverse process of absorption. By measuring the intensity of light-emitting atoms and ions, the density of these species can be determined, in case of optically thin lines. When in LTE, the ASDFs can be constructed from the density; these obey the distribution function as described by Boltzmann and Saha. 4

Another aspect of LTE is that the kinetic temperature of the electrons is the same as that of the heavy particles, i.e., $T_{e}=T_{h}$.

Figure 3 gives a sketch of the ASDF in LTE of two subsequent systems. Such a graph is expected for the ASDF of the atomic and ionic systems of the additive in the lamp. It can be expressed by the Saha equation ${ }^{5}$

$$
\eta_{p}=\eta_{e} \eta_{r}^{+}\left(\frac{h^{2}}{2 \pi m_{e} k_{b} T_{e}}\right)^{3 / 2} \exp \left(I_{p r} / k_{B} T_{e}\right)
$$

where $m_{e}, k_{B}$, and $T_{e}$, are the mass of the electron, the Boltzmann constant and the electron temperature, respectively. $I_{p r}$ is the energy difference between atomic level $p$ and ionic level $r$. In the case of $r=1$, which is the ground level of the ionic system, we have $I_{p 1} \equiv I_{p}$, where $\mathrm{I}_{p}$ is the ionization energy. $\eta_{\mathrm{p}}$ is the corresponding state density defined as $\eta$ $=n / g$, the number density $n$ of an atom in level $p$ divided by the statistical weight $g$ of that level. In the same way we can define $\eta_{r}^{+}=n_{r}^{+} / g_{r}^{+}$, the state density of the ion and the state density of the free electron $\eta_{e}=n_{e} / g_{e}$, where $g_{e}=2$, the number of the spin states of the free electron. When we use the Saha equation to find the relation between the atomic and ionic ground states in Dy we need to insert the ionization potential of the atomic Dy, $I_{p}=5.93 \mathrm{eV}$. 
Two atomic levels that are in the Saha relation with the ionic ground state are related to each other by Boltzmann's law (see Fig. 3). This can be understood by applying the Saha relation to two levels, $p$ and $q$, and dividing the obtained expressions. This gives

$$
\eta_{q}=\eta_{p} \exp \left(-E_{p q} / k_{B} T_{e}\right),
$$

where $E_{p q}=I_{p}-I_{q}$ (see Fig. 3). When the whole ASDF is consistent with Saha (and thus with Boltzmann), the plasma region is said to be in local thermal equilibrium. ${ }^{5}$

We study the various features of the ASDF as shown in Fig. 3 with the aim to deduce essential plasma properties from it. Since Fig. 3 is a semilog plot, it is instructive to take the logarithm of the corresponding equation, i.e., the Saha equation (3) which gives

$$
\ln \eta_{p}=\frac{I_{p}}{k_{B} T_{e}}+\ln \left(\eta_{\infty}\right),
$$

where

$$
\eta_{\infty}=\eta_{e} \eta_{1}^{+}\left(\frac{h^{2}}{2 \pi m_{e} k_{B} T_{e}}\right)^{3 / 2} .
$$

This near-continuum value of the atomic state density can be obtained by extrapolating $\eta_{p}$ towards $\mathrm{I}_{p}=0$. Other important densities are the atomic ground state density $\eta_{1}$ and the ionic ground state density $\eta_{1}^{+}$. These can be obtained by extrapolating the ASDF towards $\mathrm{E}_{1 p}=0$ and $\mathrm{I}_{p 1}=0$.

The total ion density is found by taking the sum of all (excited) ions using the following expression:

$$
n^{+}=\sum_{q} n_{q}^{+},
$$

where

$$
n_{q}^{+}=\eta_{1}^{+} g_{q} \exp \left(\frac{-E_{q}}{k_{B} T}\right),
$$

where $\eta_{1}^{+}$is the ionic ground state density. Rewriting Eq. (7) gives

$$
n^{+}=\eta_{1}^{+} \sum_{q} g_{q} \exp \left(\frac{-E_{q}}{k_{B} T}\right)=\eta_{1}^{+} Z(T) .
$$

$Z(T)$ is the partition function depending only on temperature and considered species. ${ }^{4}$ The total atomic density of Dy can be calculated by means of the same expression in which the ionic ground state density is replaced by that of the atom and the partition function for the atom is used. In contrast to the $\mathrm{Hg}$ system, where we can replace the partition function by the $g$ value of the ground level $\left(g_{1}=2\right)$ without loss of accuracy, the partition function must be taken into account for the Dy system. For instance, the value for $Z(T)$ for Dy ions at $T=5900 \mathrm{~K}$ is 69 and for Dy atoms is $59,{ }^{6}$ whereas $g_{1}$ of Dy atoms and $g_{1}$ of Dy ions are 17 and 18, respectively.

We now introduce step by step how to extract information from the semilog plot of Fig. 3.

(1) The temperature value can be determined from the slope of the ASDF. This temperature can be indictated as the excitation temperature $T_{\mathrm{exc}}$, which in LTE should be the same as the electron temperature $T_{e}$ and the heavy particle temperature $T_{h}$ (see Fig. 3). Since we construct an ASDF for different species, namely, $\mathrm{Hg}$ atoms and Dy atoms and ions, we will find $T_{\text {exc }}$ values for each of these species. If the temperatures are equal within the estimated error margins, one of the necessary demands of the LTE assumption is satisfied.

(2) By extrapolating the line in the atomic system toward the ground state (that is, $\mathrm{E}_{1 p}=0$ ), we find the value of the atomic ground state density. As we can only measure the visible spectrum we cannot measure lines from low energy levels; therefore extrapolation is necessary.

(3) By extrapolating the line in the atomic system toward the continuum (that is, $\mathrm{I}_{p}=0$ ), we find the value of $\eta_{\infty}$. By extrapolating the line in the ionic system toward the continuum, we find the value of the ionic ground state $\eta_{1}^{+}$(see Fig. 3).

(4) The electron density $n_{e}$ can be calculated from the Saha jump, which is the difference between the occupation of the highly excited state $\eta_{\infty}$ and the ionic ground state $\eta_{1}^{+}$. By determining $\eta_{\infty}$ and $\eta_{1}^{+}$(both by extrapolation) and $\mathrm{T}_{e}$ (from the slope), $n_{e}$ can be calculated.

We recall that in a mixture, such as the composition of $\mathrm{Hg}$ and $\mathrm{DyI}_{3}$, the electrons present are supplied by both $\mathrm{Hg}$ and Dy, and are indistinguishable. Due to charge neutrality we may expect that the relation $n_{e}=n_{i}(\mathrm{Hg})+n_{i}(\mathrm{Dy})$ holds. It will be shown that $\mathrm{Hg}$ is dominant in the lamp we studied.

The ionic ground state densities of Dy is determined with two different methods. One method (the "Boltzmann method") calculates the ionic ground state density from the extrapolation of the ionic Dy lines in the Boltzmann plot to the ionization potential [see Eq. (3)] The other method (the "Saha method") combines the value for $\eta_{\infty}$ from the atomic Dy system with the electron density calculated using $\mathrm{Hg}$ (assuming $n_{i}=n_{e}$ ).

\section{B. The absolute line intensity}

In order to construct an ASDF we need to relate the intensity of Dy and $\mathrm{Hg}$ lines measured in the experiment to the absolute densities. If the plasma is optically thin, the radiation can escape the plasma, as absorption can be neglected. The corresponding integrated emission coefficient of a particular transition $j_{p q}\left[\mathrm{~W} \mathrm{~m}^{-3} \mathrm{sr}^{-1}\right]$, is defined as

$$
j_{p q}=(1 / 4 \pi) n_{p} A_{p q} E_{p q},
$$

where $n_{p}$ is the density of the upper level $p, A_{p q}$ is the transition probability, $E_{p q}=E_{1 p}-E_{1 q}=h \nu_{p q}$ is the energy of the emitted photon. This equation shows that the population density $n_{p}$ of level $p$ is proportional to the integrated emission coefficient $j_{p q}$,

$$
n_{p}=4 \pi \frac{j_{p q}}{A_{p q} E_{p q}} .
$$

The calibrated intensity $I_{p q}\left[\mathrm{~W} \mathrm{~m}^{-2} \mathrm{sr}^{-1}\right]$ is determined by calibrating the line intensity of the metal-halide lamp with a tungsten ribbon lamp at the line-of-sight over a plasma column of length $D$. It is given by 


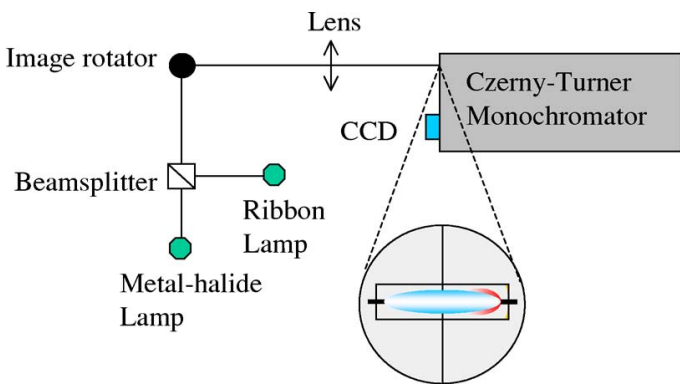

FIG. 4. (Color online) Setup consisting of a Czerny-Turner $1 \mathrm{~m}$ monochromator, an image rotator, a beam splitter, and a ST-2000 CCD camera. The $\mathrm{CCD}$ camera records the $2 \mathrm{D}$ image of the light after being dispersed into different wavelengths by the grating of the monochromator. The wavelengths of the various atomic and ionic transitions are found on the horizontal scale. The image rotator optically rotates the image of the burner by $90^{\circ}$ so it is projected horizontally onto the entrance slit, yielding a CCD image of the cross section of the burner on the vertical scale.

$$
I_{p q}=\int_{0}^{D} j_{p q} d s
$$

$j_{p q}$ is not necessarily constant over the length of the column, but could vary over the radial distance. Line intensities are measured at the line of sight. This means that the intensity of the spectral line is measured laterally, which is in our case along the horizontal cross section of the burner. A lateral profile constitutes the integrated spectral line intensity as a function of the lateral position. Radial information can be extracted from a set of lateral measurements by the Abel inversion technique. ${ }^{7}$ The radial intensity profile $j_{p q}$ is approximated by means of a polynomial series, ${ }^{8}$

$$
j_{p q}(r)=a_{0}+\sum_{n=2}^{\infty} a_{n} r^{n} .
$$

The term $a_{1} r$ is omitted because the derivative of the dysprosium density at the axis of the burner should be zero due to symmetry. Applying a least squares fitting procedure to the lateral profile, the coefficients $a_{n}$ are obtained and a radial profile is constructed.

After selecting a number of Dy and $\mathrm{Hg}$ lines from the measured spectrum of $400-800 \mathrm{~nm}$, based on Ref. 9, the lateral profile of the corresponding emitting species can be constructed. The radial profiles are then used to construct the ASDF for several radial positions. Measurements were done at different axial positions in the lamp. the results reported here were found at $3 \mathrm{~mm}$ above the bottom electrode with a $50 \mu \mathrm{m}$ resolution in height.

\section{EXPERIMENTAL SETUP}

In the present experiment, emission spectroscopy is performed on a metal-halide lamp. The lamp consists of a quartz burner of $20 \mathrm{~mm}$ length and $8 \mathrm{~mm}$ inner diameter and a transparent low-expansion quartz vacuum jacket. The burner is made of quartz in order to make the arc optically accessible. Because quartz does not withstand high temperatures, the chosen power is low, causing less of the additives to be present in the arc vapor than in commercially available lamps. The distance between the electrodes is approximately

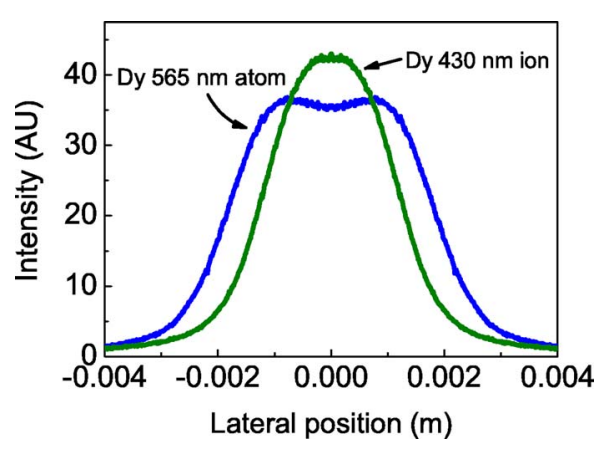

FIG. 5. (Color online) Lateral intensity profiles of the atomic (at $565 \mathrm{~nm}$ ) and ionic (at $430 \mathrm{~nm}$ ) Dy. The profiles are symmetrized with regard to the axis of the arc. The lines are measured $3 \mathrm{~mm}$ above the bottom electrode; the lamp power is $100 \mathrm{~W}$.

$18 \mathrm{~mm}$. The lamp is dosed with $10.0 \mathrm{mg}$ of $\mathrm{Hg}$ and $4.08 \mathrm{mg}$ of $\mathrm{DyI}_{3}$. It is driven by a ballast with an $80 \mathrm{~Hz}$ square wave voltage and operated with an average input power of $100 \mathrm{~W}$.

The metal-halide lamp ${ }^{10}$ is mounted on an optical rail in vertical position, together with a beam splitter, an image rotator, and a lens (with a focal length of $200 \mathrm{~mm}$ ), which focuses the lamp image onto the entrance slit of the spectrometer (see Fig. 4). The Czerny-Turner $1 \mathrm{~m}$ monochromator contains a 1200 lines $/ \mathrm{mm}$ grating for wavelength separation and an ST-2000 charge-coupled device (CCD) camera for two-dimensional (2D) imaging. This CCD chip is 1600 pixels wide and 1200 pixels high. The pixel size is $7.4 \times 7.4 \mu \mathrm{m}^{2}$. The image rotator rotates the lamp image by $90^{\circ}$ so that a horizontal cross section of the lamp is imaged onto the vertical slit of the monochromator and then on the CCD camera. As the wavelength dispersion is in the horizontal direction, we obtain, at one axial position, a complete lateral profile in one measurement. The two-dimensional CCD image therefore contains in the vertical direction the cross section of the lamp and in the horizontal direction the wavelengths of different atomic and ionic transitions. The intensity of the Dy and $\mathrm{Hg}$ lines are calibrated with a tungsten ribbon lamp with known spectral intensity. The ribbon lamp is placed such that its calibrated light follows an optical path that is almost identical to that of the metal-halide lamp.

\section{RESULTS AND DISCUSSION}

Because the lateral profiles are not entirely symmetrical and a cylinder symmetry is assumed, the lateral profiles are

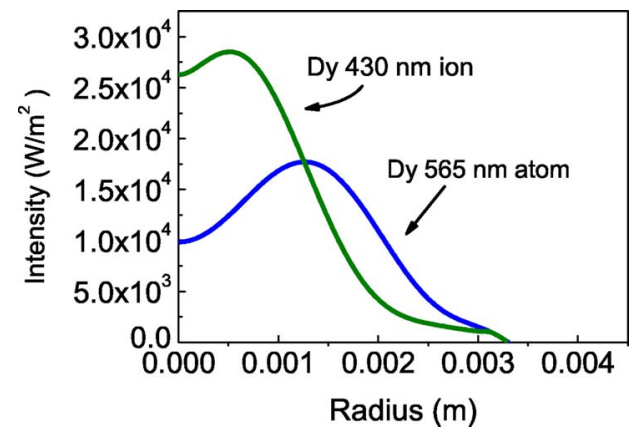

FIG. 6. (Color online) Calibrated radial intensity profiles of the atomic (at $565 \mathrm{~nm}$ ) and ionic (at $430 \mathrm{~nm}$ ) Dy. The lines are measured $3 \mathrm{~mm}$ above the bottom electrode; the lamp power is $100 \mathrm{~W}$. 


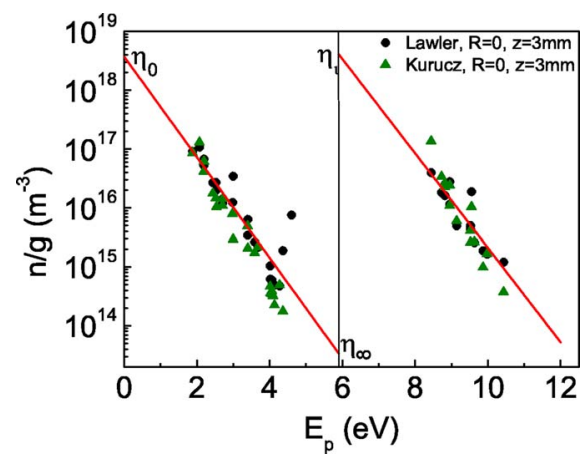

FIG. 7. (Color online) ASDF for Dy in the wavelength range of 400-700 $\mathrm{nm}$. Emission lines are measured at the center of the lamp $3 \mathrm{~mm}$ above the electrode. These results have been Abel inverted. The atomic and ionic transition probabilities are taken from Wickliffe and Lawler ${ }^{11}$ and Kurucz and Bell (Ref. 9).

symmetrized with regard to the axis of the arc. The temperature at the axis is highest, and the axis can therefore be found there where the intensity of $\mathrm{Hg}$ is highest. An example of the symmetrized lateral profiles in Fig. 5 shows the different lateral distributions of atomic and ionic species of Dy. Ions are at the center, surrounded by atoms, which are in turn surrounded by (invisible) molecules near the wall. This is even more evident when looking at the radial intensity profiles in Fig. 6 derived from Fig. 5 using the previously described Abel inversion technique. There is a small dip in the center of the ionic Dy; this is most likely a temperature effect, as the temperature in the center of the lamp is highest. It may also be caused by the Abel inversion technique, which is very sensitive for errors in the center of the lateral profile.

From the radial profiles the ASDFs can be constructed for different radial positions. Figure 7 shows the ASDF at the axis of the lamp, $3 \mathrm{~mm}$ above the bottom electrode. Transition probabilities $A$ from two different sources, Wickliffe and Lawler ${ }^{11}$ and Kurucz and Bell, ${ }^{9}$ were used. The measured lines and corresponding $A$ values and the relative errors are listed in the Appendix

The temperature of the atomic Dy using the Lawler values is $5900 \mathrm{~K}$, and that of the ionic Dy is $6300 \mathrm{~K}$. The temperature of the atomic Dy using the Kurucz values is $4700 \mathrm{~K}$, and that of the ionic Dy is $4400 \mathrm{~K}$. The Lawler values for $A$ were found using an experimental technique that

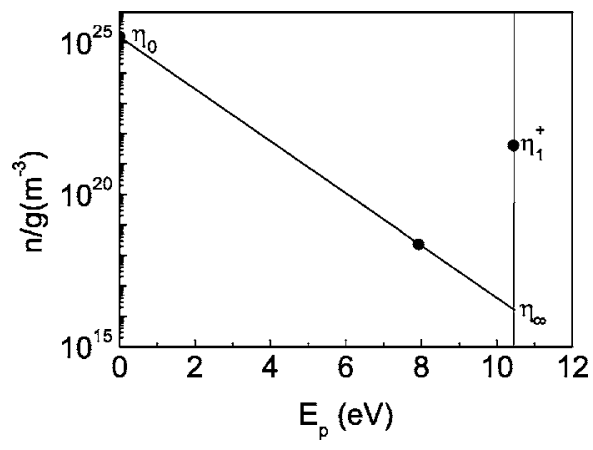

FIG. 8. ASDF for $\mathrm{Hg}$ emission lines that are measured at the center of the lamp $3 \mathrm{~mm}$ above the electrode. The ionization potential of $\mathrm{Hg}$ is at 10.44 $\mathrm{eV}$. The ground state density is calculated from $p=n k T$. The intensity profile of the $407.8 \mathrm{~nm}$ line, with $A=4 \times 10^{6}$ (Ref. 11), at $E_{p}=7.92 \mathrm{eV}$ has been Abel inverted.

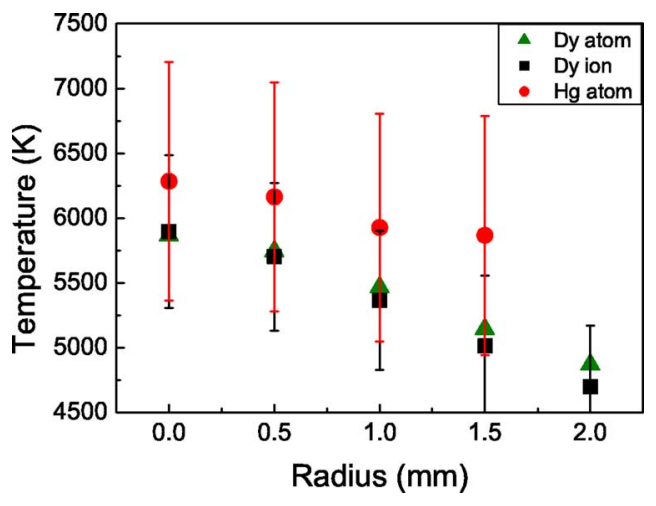

FIG. 9. (Color online) Temperature profiles for different radial positions 3 $\mathrm{mm}$ above the bottom electrode. The lamp power is $100 \mathrm{~W}$. The transition probabilities were taken from Wickliffe and Lawler (Ref. 11).

consists of a combination of fluorescent lifetime measurements of different levels and a determination of branching fractions by fourier transform spectroscopy. The values of Kurucz were determined by the estimation of the line intensity. Further results described in this paper make use of the Lawler values for $A$ as we assume that the method used to determine them is more reliable. It is clear, however, that the errors in the $A$ values are a strong limitation for the interpretation of our measurements. From the ASDF at different radial positions a radial temperature profile can be constructed for the ionic and atomic Dy.

We now examine the $\mathrm{Hg}$ system. Only a small number of atomic $\mathrm{Hg}$ lines were observed, of which the energy levels were very close so that no accurate slope of the ASDF could be determined. This can be resolved by taking the absolute density of the ground state into account. First we calculate the ground state density of the $\mathrm{Hg}$ atom using the ideal gas law $p=n k T$. Due to the $\mathrm{Hg}$ dominance, $n$ can be replaced by the $n_{\mathrm{Hg}}$ density. As the pressure is constant over the lamp, $n T$ is a constant over the whole plasma. We determine the pressure by calculating the total density of $\mathrm{Hg}$ inside the burner. This is done using the dimensions and the $\mathrm{Hg}$ content of the lamp, which is $20 \mathrm{~mm}$ in height and $8 \mathrm{~mm}$ in diameter and contains $10 \mathrm{mg}$ of $\mathrm{Hg}$, together with the assumption that the effective temperature equals $3000 \mathrm{~K}{ }^{12}$ We then assume a LTE and use the temperature profile for the atomic Dy in combination with the calculated pressure to find the ground

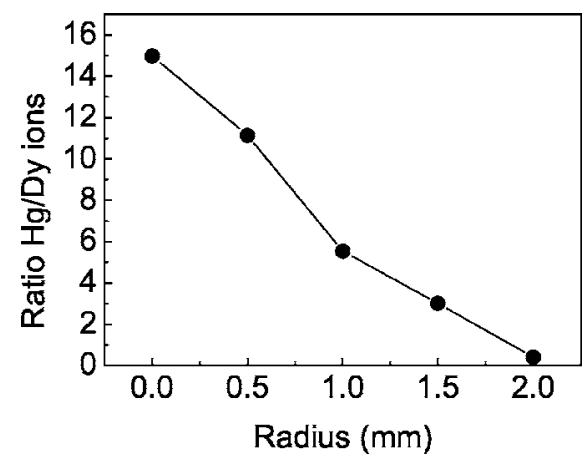

FIG. 10. The ratio of $\mathrm{Hg} / \mathrm{Dy}$ ions for different radial positions. The total ionic density $n^{+}$is calculated by combining the expression for $\eta_{\infty}$ [Eq. (6)] with the expression for $n^{+}$[Eq. (9)]. $\eta_{\infty}$ is found by means of the extrapolation of the ASDF of Dy and $\mathrm{Hg}$ at different radial positions. 


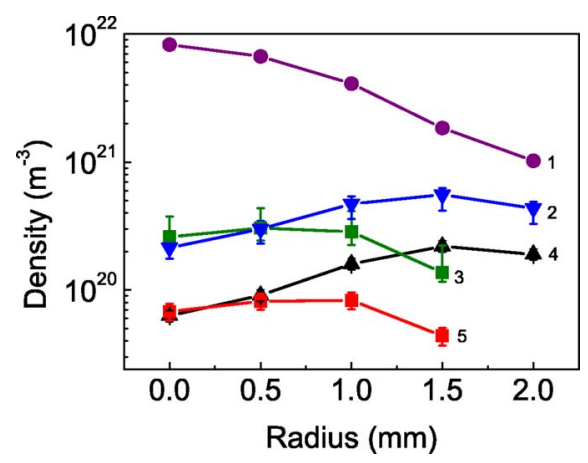

FIG. 11. (Color online) (1) electron density determined from the Hg ASDF. Total atomic (2) and ionic densities (3) for Dy. Atomic (4) and ionic (5) ground state densities for Dy.

state density of a $\mathrm{Hg}$ as function of the radius. It must be stated that the initial choice of temperature has a limited effect on the results. In order to construct the ASDF, we determine the absolute density at one line, at $407.8 \mathrm{~nm}$ (see Fig. 8). We can now calculate the temperature as a function of the radius.

Figure 9 shows that the temperature profiles for the atomic and the atomic ionic Dy and $\mathrm{Hg}$ are similar when taking the error of the linear fit of the scattered data points into account which, due to the large scatter of the data points, is relatively high and about $10 \%-14 \%$.

Now that the temperature is known we calculate the ground states of atoms and ions as well as the electron density and the total atomic and ionic density of Dy. From Figs. 7 and 8 it can be deduced that $\mathrm{Hg}$ is the dominant ion. Therefore the ratio between the $\mathrm{Hg}$ ion and the Dy ion has been calculated from these measurements. The ratio of $\mathrm{Hg} / \mathrm{Dy}$ ions is shown as a function of the radius in Fig. 10, proving that $\mathrm{Hg}$ ions dominate over the Dy ions up to $R=1.7 \mathrm{~mm}$. Because $\mathrm{Hg}$ ions are the leading system in the center of the lamp, the bulk of the electrons are supplied by Hg. The electron density $n_{e}$ was determined by calculating $\eta_{\infty}$ by means of extrapolating the atomic $\mathrm{Hg} \mathrm{ASDF}$ and assuming $n_{i}=n_{e}$ for the inner region. The results are shown in Fig. 11.

Total atomic and ionic densities are also shown in Fig. 11. Dy atoms ionize into Dy ions in the hot center of the lamp, causing a depletion of Dy atoms. The atomic density decreases toward the cooler wall, making room for mol-

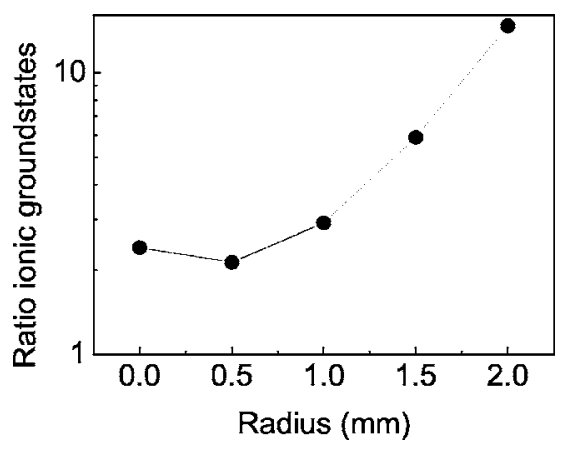

FIG. 12. The ratio between the ionic ground state density calculated using the value for $\eta_{\infty}$, combined with the electron density calculated using the $\mathrm{Hg}$ density (assuming $n_{i}=n_{e}$ ), and the ionic ground state density calculated from the extrapolation of the ASDF for the Dy ion. ecules. The atomic ground state density found by extrapolating the atomic ASDF is of the order $n_{1}=10^{20} \mathrm{~m}^{-3}$ (taking $g_{1}=17$ ). Flikweert et al. ${ }^{8}$ measured the atomic ground state density at different powers (at 113 and $151 \mathrm{~W}$ ) by means of a laser absorption spectroscopy of a lamp of the same type. It was found that the value for the atomic ground state rapidly increases as a function of power. An extrapolation to $100 \mathrm{~W}$ from these results leads to a similar value for the atomic ground state density.

The ratio between the Saha method and the Boltzmann method is depicted in Fig. 12. It shows that when moving away from the center we see an increasing discrepancy between the two extrapolated ionic ground state densities. The ionic system is underpopulated with respect to the atomic system. This is what we expect as the ionic system suffers more loss of radiation than does the atomic. This means that the Boltzmann balance of excitation and deexcitation is no longer in equilibrium and that the assumption of a LTE is no longer valid.

\section{CONCLUSIONS}

A radially resolved ASDF has been constructed for Dy and $\mathrm{Hg}$ for the metal-halide lamp. Radial intensity profiles of Dy show a clear separation between atomic and ionic regions in the plasma. The ionic region is in the hot center; the atomic region surrounds it. The radial temperature profile

TABLE I. $A$ values used for the calculation of the density of the atomic Dy. $A$ values and relative errors are from Wickliffe and Lawler (Ref. 11). Values from Kurucz and Bell (Ref. 9) are based on data provided by either Gorshkov et al. (Ref. 13), where the relative error is estimated to be less than $50 \%$, or by Meggers et al. (Ref. 14), from which the $A$ values are determined from the estimated intensity.

\begin{tabular}{ccc}
\hline \hline $\begin{array}{c}\text { Line } \\
(\mathrm{nm})\end{array}$ & $\begin{array}{c}A \text { value Lawler } \\
\left(\times 10^{6} \mathrm{~s}^{-1}\right)\end{array}$ & $\begin{array}{c}A \text { value Kurucz } \\
\left(\times 10^{6} \mathrm{~s}^{-1}\right)\end{array}$ \\
\hline 402.371 & $29.0 \pm 5 \%$ & 58.55 \\
411.305 & $23.4 \pm 5 \%$ & 92.10 \\
412.608 & $0.92 \pm 5 \%$ & 73.1 \\
413.035 & $1.76 \pm 5 \%$ & 1.84 \\
414.606 & $195.0 \pm 5 \%$ & 347.8 \\
419.802 & $108.0 \pm 5 \%$ & 214 \\
423.202 & $79.0 \pm 5 \%$ & 146.8 \\
423.985 & $94.0 \pm 5 \%$ & 145 \\
424.591 & $118.0 \pm 5 \%$ & 178.9 \\
429.196 & $2.28 \pm 5 \%$ & 6.159 \\
448.436 & $4.65 \pm 6 \%$ & 10.48 \\
456.509 & $0.66 \pm 5 \%$ & 0.864 \\
457.778 & $1.96 \pm 5 \%$ & 2.448 \\
488.016 & $0.520 \pm 5 \%$ & 0.7924 \\
488.808 & $4.97 \pm 5 \%$ & 6.098 \\
502.212 & $1.27 \pm 5 \%$ & 1.504 \\
504.264 & $6.9 \pm 5 \%$ & 6.065 \\
507.067 & $4.59 \pm 5 \%$ & 6.303 \\
507.767 & $0.41 \pm 8 \%$ & 0.621 \\
563.950 & $0.49 \pm 8 \%$ & 0.6007 \\
565.201 & $0.446 \pm 5 \%$ & 0.4491 \\
598.856 & $0.561 \pm 5 \%$ & 0.4636 \\
616.843 & $0.81 \pm 5 \%$ & 1.702 \\
657.937 & $0.77 \pm 10 \%$ & 0.8212 \\
\hline \hline
\end{tabular}


TABLE II. $A$ values used for the calculation of the density of the ionic Dy. $A$ values and relative errors are from Wickliffe and Lawler (Ref. 11). Values from Kurucz and Bell (Ref. 9) are based on data provided by either Gorshkov et al. (Ref. 13), where the relative error is estimated to be less than $50 \%$, or by Meggers et al. (Ref. 14), from which the $A$ values are determined from the estimated intensity.

\begin{tabular}{ccc}
\hline \hline $\begin{array}{c}\text { Line } \\
(\mathrm{nm})\end{array}$ & $\begin{array}{c}A \text { value Lawler } \\
\left(\times 10^{6} \mathrm{~s}^{-1}\right)\end{array}$ & $\begin{array}{c}A \text { value Kurucz } \\
\left(\times 10^{6} \mathrm{~s}^{-1}\right)\end{array}$ \\
\hline 395.039 & $25 \pm 6 \%$ & 53.90 \\
397.856 & $49.9 \pm 5 \%$ & 68.91 \\
399.669 & $16.3 \pm 6 \%$ & 18.51 \\
400.045 & $25.4 \pm 5 \%$ & 23.63 \\
403.633 & $1.73 \pm 8 \%$ & 3.563 \\
407.312 & $12.1 \pm 7 \%$ & 20.09 \\
411.134 & $2.84 \pm 5 \%$ & 2.983 \\
412.463 & $7.2 \pm 5 \%$ & 11.77 \\
414.310 & $8.1 \pm 8 \%$ & 9.82 \\
425.633 & $0.79 \pm 5 \%$ & 0.9048 \\
430.863 & $2.78 \pm 5 \%$ & 1.71 \\
444.970 & $1.97 \pm 5 \%$ & 1.062 \\
446.814 & $0.451 \pm 5 \%$ & 0.4441 \\
495.735 & $1.38 \pm 5 \%$ & 0.4011 \\
\hline \hline
\end{tabular}

constructed from the ASDF of the atomic Dy shows a temperature of $5900 \mathrm{~K}$ in the center of the lamp. The ionic and atomic Dy temperature profile and the atomic $\mathrm{Hg}$ temperature profile are all equal within the error margins. Error margins are about $10 \%$ due to the large scatter in the data points. This can be remedied with better $A$ values.

In this lamp driven at $100 \mathrm{~W}, \mathrm{Hg}$ ions predominate in the center of the discharge and contribute largely to the total electron density of the discharge. At the center of the lamp there seems to be a LTE; toward the wall there is a deviation of a LTE with respect to the population of the Dy ionic system.
Future studies should include the measurement of the density distributions at different axial positions.

\section{ACKNOWLEDGMENTS}

This research is supported by Technologiestichting STW (Project No. ETF. 6093) and COST (Project 529).

\section{APPENDIX. MEASURED LINES AND CORRESPONDING $A$ VALUES}

Table I contains the $A$ values for atomic Dy lines and Table II contains the $A$ values for ionic Dy lines. $A$ values are listed from Wickliffe and Lawler ${ }^{11}$ and Kurucz and Bell. ${ }^{9}$

${ }^{1}$ G. G. Lister, J. E. Lawler, W. P. Lapatovich, and V. A. Godyak, Rev. Mod. Phys. 76, 541 (2004).

${ }^{2}$ E. Fischer, J. Appl. Phys. 47, 2954 (1976).

${ }^{3}$ X. Zhu, Ph.D. thesis, Eindhoven University of Technology, 2005.

${ }^{4}$ M. Mitchner and C. H. Kruger, Jr., Partially Ionized Gases, 1st ed. (Wiley, New York, 1973).

${ }^{5}$ J. A. M. van der Mullen, Phys. Rep. 191, 109 (1990).

${ }^{6} \mathrm{http}: / /$ www.thespectroscopynet.com/Theory/Partition_Functions.asp

${ }^{7}$ J. J. Curry, M. Sakai, and J. E. Lawler, J. Appl. Phys. 84, 3066 (1998).

${ }^{8}$ A. J. Flikweert, T. Nimalasuriya, C. H. J. M. Groothuis, G. M. W. Kroesen, and W. W. Stoffels, J. Appl. Phys. (submitted).

${ }^{9}$ R. L. Kurucz and B. Bell, Kurucz CD-ROM No. 23, Atomic Line Data, Smithsonian Astrophysical Observatory, Cambridge, MA, 1993.

${ }^{10} \mathrm{~W}$. W. Stoffels et al., Proceedings of the XXVIIth International Conference on Phenomena in Ionized Gases, Eindhoven, 17-22 July 2005, edited by E. M. van Veldhuizen (unpublished), Paper 16-342.

${ }^{11}$ M. W. Wickliffe and J. E. Lawler, J. Quant. Spectrosc. Radiat. Transf. 66, 363 (2000)

${ }^{12} \mathrm{~W}$. Elenbaas, The High Pressure Mercury Vapour Discharge, 1st ed. (North-Holland, Amsterdam, 1951).

${ }^{13}$ V. N. Gorshkov, V. A. Komarovskii, A. L. Oserovich, N. P. Penkin, and R. Khefferlin, Opt. Spektrosk. 48, 657 (1980).

${ }^{14}$ W. F. Meggers, C. H. Corliss, B. F. Scribner, Tables of Spectral-line intensities Part 1: Arranged by Elements, 2nd ed. (National Bureau of Standards, 1975). 